\title{
Keep on Trucking
}

\section{Executive Summary}

In the past century, as energy usage rapidly increased and fossil fuel consumption and carbon emissions have increased along with it, transportation has accounted for a large share of this. With e-commerce and delivery increasing in popularity and trucks accounting for around a third of transport-related carbon emissions and $20 \%$ of the global demand for oil [1], it is important to evaluate more energy-efficient and sustainable alternatives. One example of such is using electricity powered semi-trucks instead of semi trucks fueled by diesel. Multiple companies are already in the process of producing electric semi-trucks including Freightliner and Tesla, whose electric semis are supposed to enter production this year. Transitioning to electric semi-trucks could help with both reducing the environmental impact of trucks and reducing the total operating cost in the long run.

We predicted the percentage of semis that would be electric in the next twenty years by using Markov chains. Semi-trucks were split into short-haul, regional-haul, and long-haul, and a model was created for each type of truck. Operating costs were calculated using values found in the "Truck Usage Data" and the cost of operating an electric and diesel truck per mile (\$1.26 vs $\$ 1.51$ ). Purchasing costs were based on the prices of current day cab and sleeper semi-trucks along with base prices for Tesla electric semis. The difference in costs was used to estimate the probability values of replacing diesel trucks with electric trucks. These probability values were placed into three separate transition matrices for each type of semi and were then used in calculating the number of electric cars in the next 5 and 10 years in tandem with the number of inoperable diesel semi-trucks each year. We predicted the population of inoperable diesel semi-trucks in the next 8 years with the transition matrices to calculate the number of electric cars in 20 years. Our model predicts that in 5 years, 10 years, and 20 years, electric semi-trucks will make up $27.39 \%, 69.49 \%$, and $97.77 \%$ of the number of semis, respectively.

In view of that imminent, sharp increase in electric truck use, a model was also created to determine the amount of infrastructure necessary to completely transition all American fleets. Because fully charging electric vehicle batteries is slow and inefficient, we first developed an optimal pit stop schedule for long-haul single driver trips. We took into account the variety of ranges and charging times of electric vehicles in order to do this. This strategy was used in conjunction with a Monte Carlo simulation to simulate the resource needs of a large population of trucks on five shipping corridors. Interestingly, all five revealed an optimal stopping interval of 90 minutes. Finally, another Monte Carlo simulation revealed the optimal number of charging stations per truck stop, which ranged from 14 to 29 depending on average traffic and related factors.

In order to model which of the corridors should be developed for electrification we first created an importance score based on three different factors: economic growth, environmental consideration, and total cost for installation. Economic growth was found based on money saved by the decreased idling times of DCFC chargers and total average traffic. Environmen- 
tal consideration was based off of the average number of electric vehicle policy / actions in the state. Total cost was calculated using values found from the previous part on number of charging stations and chargers needed per trucking corridor. These values were then normalized and used to rank the five corridors in part 2. The results showed that the Minneapolis to Chicago corridor should be targeted first followed by Los Angeles to San Francisco then San Antonio to New Orleans then Jacksonville to D.C. then Boston to Harrisburg. 


\section{Contents}

1 Shape Up or Ship Out 189

1.1 Defining the Problem . . . . . . . . . . . . . . . . . . . . . . 189

1.2 Assumptions . . . . . . . . . . . . . . . . . . . . . . 189

1.3 Variables Used . . . . . . . . . . . . . . . . . . . . . . . . . . . . . . . . . . . . . . . . .

1.4 Developing the Model . . . . . . . . . . . . . . . . . . . . . . . . 190

1.5 Executing the Model . . . . . . . . . . . . . . . . . . . . . . . . 193

1.6 Results and Discussion . . . . . . . . . . . . . . . . . . . . . . . . . . . . . . . . . . . . . . . . .

1.6.1 Strengths and Weaknesses .................. 194

2 In It for the Long Haul 195

2.1 Defining the Problem . . . . . . . . . . . . . . . . . . . 195

2.2 Assumptions . . . . . . . . . . . . . . . . . . . . . . . . . . . . . . . . . . . . . . . . . . . . . . . .

2.3 Variables Used . . . . . . . . . . . . . . . . . . . . . . 196

2.4 Developing the Model . . . . . . . . . . . . . . . . . . . . . 196

2.5 Executing the Model . . . . . . . . . . . . . . . . . . . . . 197

2.5.1 Optimal Stations per Shipping Corridor . . . . . . . . . . . . . 197

2.5.2 Optimal Charging Stations per Truck Stop . . . . . . . . . . . . . . . 197

2.6 Results and Discussion . . . . . . . . . . . . . . . . . . . . 198

2.6.1 Strengths and Weaknesses ................... 199

3 I Like to Move It, Move It 199

3.1 Defining the Problem . . . . . . . . . . . . . . . . . . . 199

3.2 Assumptions . . . . . . . . . . . . . . . . . . . . . . . . . . 199

3.3 Variables Used . . . . . . . . . . . . . . . . . . . . . . 200

3.4 Developing the Model . . . . . . . . . . . . . . . . . . . . . 200

3.5 Executing the Model . . . . . . . . . . . . . . . . . . . . . . 202

3.6 Results and Discussion . . . . . . . . . . . . . . . . . . . 202

3.6.1 Strengths and Weaknesses ................... 202 


\section{Global Assumptions}

G-1. There are currently 1.7 million semi trucks in operation in the United States. [2]

- Justification: It is reasonable to assume that this provided data is about the United States because there are around 2 million semi trucks on the road in the United States. [10]

\section{Shape Up or Ship Out}

\subsection{Defining the Problem}

Develop a model to predict the percentage of semi trucks that will be electric 5, 10, and 20 years from 2020 .

\subsection{Assumptions}

1-1. There are no electric semi trucks currently in operation.

- Justification: Production has started for electric semi-trucks, but they will hit the roads in 2020. [11]

1-2. The market for semi-trucks demands the production of 210,466 semi-trucks per year.

- Justification: Since the production of Class 8 trucks in 2019 was 210,466 according to Truck Production Data, 2020 MathWorks Math Modeling Challenge [17], the market is assumed to be constant.

1-3. Electric semi trucks have the same life expectancy as diesel semi-trucks (12 years).

- Justification: Since electric semi-trucks are a new invention, the average life expectancy of an electric semi-truck cannot be observationally determined yet. The life expectancy will most likely depend on the owner's willingness to replace its battery.

1-4. The total difference in cost between electric and diesel semi-trucks is proportional to the probability that a diesel semi-truck will be replaced with an electric semi-truck.

- Justification: Since there is no data on the consumers purchasing electric cars, we assumed that a greater difference in cost will make consumers replace more diesel semi-trucks with electric semi-trucks. 


\subsection{Variables Used}

\begin{tabular}{|c|c|c|c|}
\hline Symbol & Definition & Units & Value \\
\hline$Q_{e}$ & Quantity of Electric Semis Replaced from Inoperable & trucks & $\ldots$ \\
\hline$Q_{d}$ & Quantity of Diesel Semis Converted from Inoperable & trucks & $\ldots$ \\
\hline$P_{e}$ & Proportion of Electric Semi-Trucks & $\ldots$ & $\ldots$ \\
\hline$P_{S}$ & Replacement Probability (Short Haul) & $\ldots$ & $\ldots$ \\
\hline$P_{R}$ & Replacement Probability (Regional Haul) & $\ldots$ & $\ldots$ \\
\hline$P_{L}$ & Replacement Probability (Long Haul) & $\ldots$ & $\ldots$ \\
\hline$P_{0}$ & Amount of Semi Trucks in 2019 & trucks & $1,734,721$ \\
\hline
\end{tabular}

\subsection{Developing the Model}

To predict the future percentages of electric and diesel semi-trucks in the next 5, 10, and 20 years, we divided semi-trucks into three types and created a model for each: short haul, regional haul, and long haul. We created three transition matrices (one for each type of semi truck) to input into the following Markov chain to do this.

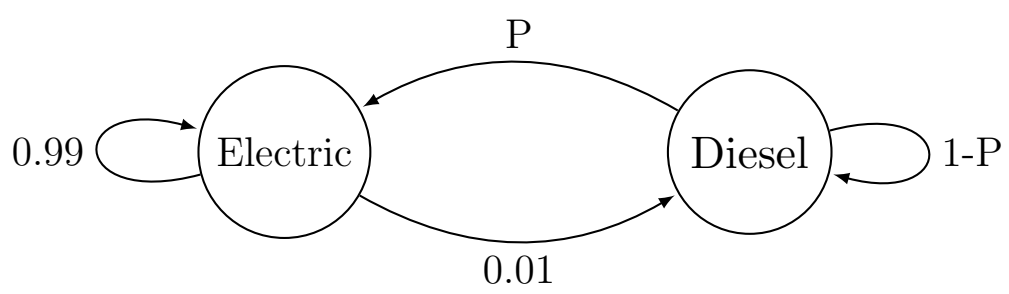

The model assumed that 0.01 of the electric cars would revert back to diesel, possibly after seeing some of the disadvantages of electric semi-trucks, including its high charge time or lower on-the-road time per refuel.

We looked into the costs of diesel and electric trucks. There were two types of diesel trucks found when researching purchase costs: day cabs, which are ideal for shorter routes and would likely be used for short and regional hauls, and sleeper trucks, which are ideal for longer routes and would likely be used for long hauls [3]. The average prices for these were found along with prices for electric semi-trucks which were based off of the base prices for the Tesla semi-trucks set to start production in 2020: $\$ 150,000$ for a 300-mile range truck (likely to be used for short hauls) and $\$ 180,000$ for a 500 -mile range truck (likely to be used for long hauls). Operating costs were then calculated and found to be $\$ 1.51$ per mile for diesel semi-trucks and $\$ 1.26$ per mile for electric semi-trucks [12].

Using those values and annual travel mileage found in "Truck Usage Data" from the 2020 MathWorks Math Modeling Challenge which was 42,640 miles for short-haul, 70,000 miles for regional, and 118,820 miles for long haul [17], the total cost over the course of 12 years (the average lifetime of a truck) and annual operating cost were calculated as shown in Table 1: 


\begin{tabular}{|c|c|c|c|c|c|}
\hline & Type & $\begin{array}{c}\text { Purchase } \\
\text { Cost }\end{array}$ & $\begin{array}{c}\text { Annual } \\
\text { Operating } \\
\text { Cost }\end{array}$ & $\begin{array}{c}\text { Total } \\
\text { Operating } \\
\text { Cost }\end{array}$ & Total Cost \\
\hline \multirow{3}{*}{ Diesel } & Short Haul & $\$ 80,000$ & $\$ 64386.40$ & $\$ 772,636.80$ & $\$ 852,636.80$ \\
& Regional & $\$ 80,000$ & $\$ 105,700$ & $\$ 1,268,400$ & $\$ 1,348,400$ \\
& Long Haul & $\$ 125,000[15]$ & $\$ 179,418.20$ & $\$ 2,153,018.40$ & $\$ 2,278,018.40$ \\
\hline \multirow{3}{*}{ Electric } & Short Haul & $\$ 150,000[16]$ & $\$ 53,726.40$ & $\$ 644,716.80$ & $\$ 794,716.80$ \\
& Regional & $\$ 150,000$ & $\$ 88,200$ & $\$ 1,050,000$ & $\$ 1,208,400$ \\
& Long Haul & $\$ 180,000[16]$ & $\$ 149,713.20$ & $\$ 1,782,300$ & $\$ 1,976,558.40$ \\
\hline
\end{tabular}

Table 1: Semi-truck Operation and Purchase Costs

With the values found in Table 1, we calculated the difference between electric semi-truck and diesel costs for purchase, operation, and total difference. In Table 2, a positive value indicates that a diesel semi-truck cost (purchase, operating, or total) is greater than the corresponding electric semi-truck cost; a negative value indicates a greater electric semi-truck cost.

\begin{tabular}{|c|c|c|c|}
\hline Type & Difference in Purchasing & Difference in Operating & Total Difference \\
\hline Short Haul & $-\$ 70,000$ & $\$ 127,920$ & $\$ 57,920$ \\
\hline Regional & $-\$ 70,000$ & $\$ 210,000$ & $\$ 140,000$ \\
\hline Long Haul & $-\$ 55,000$ & $\$ 356,460$ & $\$ 301,460$ \\
\hline
\end{tabular}

Table 2: 12-Year Difference in Costs for Diesel and Electric Semi-Trucks

The values in Table 2 were then used to calculate $\mathrm{P}$, the probability of a diesel semi-truck being replaced by an electric semi-truck in the first year, for each of the transition matrices. Short haul, regional haul, and long haul received $\mathrm{P}$ values of $0.2,0.4$, and 0.6 , respectively, due to an increase by a factor of around 2 between short haul and regional haul and regional haul and long haul total cost differences. These $\mathrm{P}$ values are then inserted into the following transition matrices to run a Markov chain model.

$$
\begin{aligned}
& \mathbf{P}_{\mathbf{S}}=\begin{array}{c||cc} 
& \multicolumn{1}{c}{D} & E \\
E & 0.8 & 0.2 \\
& 0.01 & 0.99
\end{array} \\
& \mathbf{P}_{\mathbf{R}}=\begin{array}{c||cc} 
& \multicolumn{1}{c}{D} & E \\
E & 0.6 & 0.4 \\
& 0.01 & 0.99
\end{array} \\
& \mathbf{P}_{\mathbf{L}}=\begin{array}{c||cc} 
& \multicolumn{1}{c}{D} & E \\
E & 0.4 & 0.6 \\
& 0.01 & 0.99
\end{array}
\end{aligned}
$$

These transition matrices are used to calculate the proportion of diesel and electric cars in MATLAB. 
From the Truck Production Data sheet [17], we calculated the total number of trucks produced for short-haul, regional haul, and long-haul based off of values given for total number of tractors produced and total number of long haul tractors produced. Number of short and regional haul trucks were found by subtracting the number of long hauls produced from the total. Short haul was then found by multiplying that value by $10 \%$ as short haul trucks are $5 \%$ of all semi-trucks while regional haul trucks are $45 \%$ [2] meaning that short haul trucks would account for $10 \%$ of the total of short haul and regional trucks combined.

\begin{tabular}{|c|c|c|c|}
\hline Year & $\begin{array}{c}\text { Class } 8 \\
\text { Tractor } \\
\text { Short Haul }\end{array}$ & $\begin{array}{c}\text { Class } 8 \\
\text { Tractor } \\
\text { Regional } \\
\text { Haul }\end{array}$ & $\begin{array}{c}\text { Class } 8 \\
\text { Tractor Long } \\
\text { Haul } \\
\end{array}$ \\
\hline 1999 & 3691 & 33219 & 180,205 \\
\hline 2000 & 3521.1 & 31689.9 & 105,632 \\
\hline 2001 & 2512.4 & 22611.6 & 48,771 \\
\hline 2002 & 3979.4 & 35814.6 & 62,241 \\
\hline 2003 & 4565.2 & 41086.8 & 60,516 \\
\hline 2004 & 7703.5 & 69331.5 & 77,035 \\
\hline 2005 & 7789.3 & 70103.7 & 112,091 \\
\hline 2006 & 9000.8 & 81007.2 & 114,555 \\
\hline 2007 & 3687.8 & 33190.2 & 48,884 \\
\hline 2008 & 3550.5 & 31954.5 & 57,930 \\
\hline 2009 & 2300.9 & 20708.1 & 46,715 \\
\hline 2010 & 3779.8 & 34018.2 & 44,372 \\
\hline 2011 & 7423.7 & 66813.3 & 71,325 \\
\hline 2012 & 7436.1 & 66924.9 & 80,558 \\
\hline 2013 & 7066.7 & 63600.3 & 67,896 \\
\hline 2014 & 10329.5 & 92965.5 & 74,799 \\
\hline 2015 & 10799.5 & 97195.5 & 95,769 \\
\hline 2016 & 6835.5 & 61519.5 & 55,927 \\
\hline 2017 & 6808.6 & 61277.4 & 70,866 \\
\hline 2018 & 10323.9 & 92915.1 & 91,551 \\
\hline 2019 & 10944.2 & 98497.8 & 101,024 \\
\hline
\end{tabular}

Figure 1: Production of short-haul, regional haul, and long-haul trucks

The values in Figure 1 were used to find the number of inoperable diesel semi-trucks in the next five years and the next ten years. Since the lifetime of a semi-truck is about 12 years, the semi-trucks that were produced from 2008-2012 will be inoperable in the next five years, the sum of these would be the number of inoperable trucks by the next five years that could be potentially replaced by electric trucks.

To find the number of electric and diesel cars in the next twenty years we predicted the amount of inoperable diesel semi-trucks from 2020-2027 with the assumption of a constant demand of 210,466 semi-trucks per year. 


\subsection{Executing the Model}

Using the Markov chain and the three different transformation matrices (for short, regional, and long haul) above, we were able to forecast the number of electric semi-trucks and the number of diesel semi-trucks in use in 5, 10, and 20 years from 2020 by using MATLAB. We did this by simulating Markov chains with an initial condition of all diesel semi-trucks. We can calculate the amount of electric and diesel semi-trucks in a given year by multiplying the probability of a semi-truck being electric or diesel by the amount of inoperable trucks in that year. This is calculated by using semi-truck production data from 12 years (the life expectancy of a semi-truck) prior to the forecasted year.

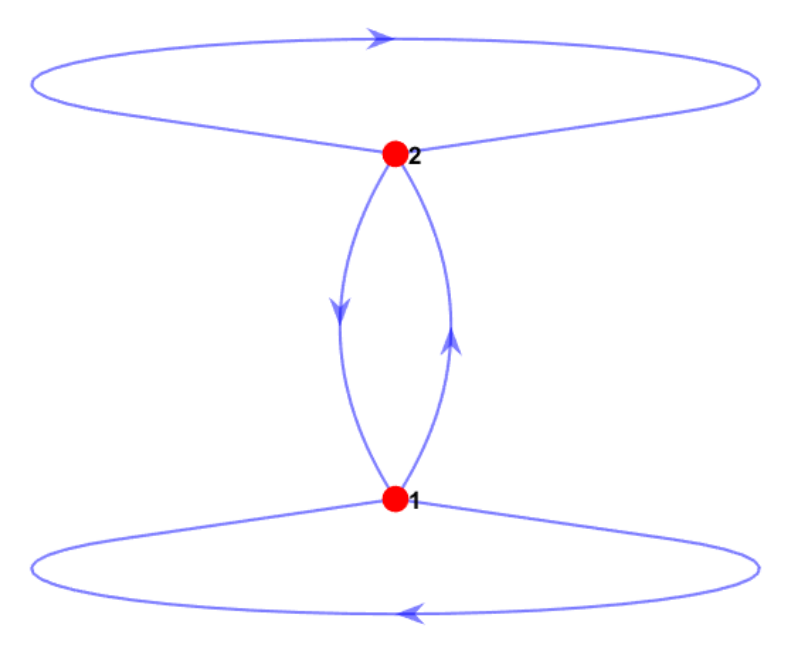

Figure 2: Markov chain produced in MATLAB

\begin{tabular}{|c|c|c|c|c|}
\hline & Short Haul & Regional Haul & Long Haul & Total \\
\hline 5 years & 12,577 & 170,234 & 261,829 & 444,640 \\
\hline 10 years & 45,945 & 530,515 & 620,694 & $1,197,154$ \\
\hline 20 years & 64,849 & 716,815 & 810,108 & $1,591,772$ \\
\hline
\end{tabular}

Table 3: Quantity of Electric Semi-Trucks Replaced from Inoperable Semi-Trucks, $Q_{e}$ (rounded down to the nearest whole truck)

\begin{tabular}{|c|c|c|c|c|}
\hline & Short Haul & Regional Haul & Long Haul & Total \\
\hline 5 years & 11,913 & 50,184 & 39,070 & 101,167 \\
\hline 10 years & 20,385 & 66,461 & 45,462 & 132,308 \\
\hline 20 years & 22,749 & 71,574 & 48,623 & 142,946 \\
\hline
\end{tabular}

Table 4: Quantity of Diesel Semi-Trucks Replaced from Inoperable Semi-Trucks, $Q_{d}$ (rounded down to the nearest whole truck)

To determine the proportion of semi-trucks that will be electric 5, 10, and 20 years from 2020, we then take the quotient of the quantity of electric semi-trucks and the total number 
of semi-trucks, which is found by analyzing the production data of diesel trucks of the past 12 years, assuming that a semi-truck is used immediately after its production:

$$
P_{e}=\frac{Q_{e}}{P_{0}}
$$

The resulting proportions of electric semi-trucks for each type of haul in 5, 10, and 20 years from 2020 are shown in Table 5.

\begin{tabular}{|c|c|}
\hline & Proportion \\
\hline 5 years & 0.2563 \\
\hline 10 years & 0.6901 \\
\hline 20 years & 0.9176 \\
\hline
\end{tabular}

Table 5: Proportion of Electric Semi-Trucks, $P_{e}$ (rounded)

\subsection{Results and Discussion}

Seeing as the total cost difference of long haul semi-trucks was the greatest, our assumption that this difference is proportional to the probability of changing to electric resulted in the largest proportion of electric trucks being long haul. Short-haul electric semi-trucks had the smallest total difference in cost between diesel short-haul semi-trucks, but the growth was still substantive. The growth of the diesel semi-trucks was steadily increasing, and the short haul diesel semi-trucks almost doubled from 5 years to 20 years in trucks due to its smaller $\mathrm{P}$ value. The totals of the quantities of semi-trucks replaced from inoperable semi-trucks after 20 years add up to the population if the trucks were not rounded down to the nearest whole truck.

\subsubsection{Strengths and Weaknesses}

The Markov chain model allows for multiple factors to be accounted for in determining the amount of electric cars at a given year after 2019. The model accounts for all of the factors given to us and includes an extra factor in the type of semi-truck. The model accounts for the annual new production rates and life expectancy by using the values 12 years prior to the predicted year in calculating how many inoperable diesel cars are expected to be replaced by electric cars. It accounts for the cost difference between electric and diesel semi-trucks by comparing the total difference between the three types of semi-trucks when determining the probability of converting an inoperable diesel semi-truck to an electric semi-truck. The model implicitly accounts for the current fleet of operational semi-trucks.

The Markov chain model does have a weakness in its determination of probability values for different types of semi-trucks. Once the trucks are released to the market, the values for these can be determined on the basis empirical data. It is also weak in its inability to determine the amount of electric semi-trucks that will replace operable diesel semi-trucks. This can be factored into the model once 2020 data is released for this purpose. The model fails to take hybrid cars into account, but the Markov chain can be modified to include a 
third state of hybrid vehicles.

Another weakness of the model is the assumption that the market for semi-truck demand is constant. In a real-world situation, the demand would fluctuate based on a variety of factors, such as pricing, competition, and battery improvements. Using a Markov Chain Monte Carlo to take these factors into account would result in more accurate $\mathrm{P}$ values based on market conditions to put into the transition matrices.

\section{In It for the Long Haul}

\subsection{Defining the Problem}

Even if all shipping companies switched over to electric semis tomorrow, the infrastructure necessary for making electric semis feasible still requires significant capital investment. The purpose of this model is to determine the most effective distribution of Electric Vehicle Supply Equipment (EVSE) along common shipping corridors in terms of money and time saved.

In order to answer this, we will also find the best charging schedule for drivers.

\subsection{Assumptions}

2-1. The state of charge of a $\mathrm{LiFePO}_{4}$ rechargeable battery exponentially approaches $100 \%$.

- Justification: Rechargeable batteries charge similarly to capacitors in that the amount of charge determines the charging speed. The more charge in the battery, the slower more charge will enter. This creates a charge over time relation proportional to $1-e^{-k x}[13][14]$.

2-2. The range of a semi truck is directly proportional to its state of charge.

- Justification: Batteries emit a constant voltage.

2-3. All EVSEs use Direct Current Fast Charging hardware, which greatly decreases charge time.

- Justification: We will prove this is optimal in Part 3.

2-4. Semi-trucks will look to recharge upon reaching a state of $20 \%$ or less.

- Justification: This reduces both risk of running empty and drivers' range anxiety [4]. 


\subsection{Variables Used}

\begin{tabular}{|c|c|c|c|}
\hline Symbol & Definition & Units & Value \\
\hline$I$ & Idle Time at Charging Station & Minutes & $\ldots$ \\
\hline$C_{0}$ & Initial State of Charge $[0,1]$ & N/A & $20 \%$ \\
\hline$C_{f}$ & Final State of Charge $[0,1]$ & N/A & $\ldots$ \\
\hline$R$ & Range & Miles & $\ldots$ \\
\hline$D$ & Distance & Miles & $\ldots$ \\
\hline$t_{80}$ & Charge Time to $80 \%$ & Minutes & $\ldots$ \\
\hline$I_{\min }$ & Minimum Time at Charging Station & Minutes & 10 \\
\hline$T_{t o t}$ & Annual Average Daily Truck Traffic & Trucks/Day & $\ldots$ \\
\hline
\end{tabular}

\subsection{Developing the Model}

Ultimately, the number of stations along a given route will depend on how often the truck needs to stop to recharge. The time taken to charge, however, must be carefully considered. Because of Assumption 2-1, it would be a waste of time to charge to $100 \%$ every time; simple algebra shows that with exponential approach, it would take the same amount of time to charge the first $70 \%$ that it would to charge the final $30 \%$.

In order to quantify this, we created a model to find the optimal charging value, $C_{f}$, that minimizes the total charging time, $I$, on a trip given the range of the truck $R$, the trip distance $D$, and the $80 \%$ recharging time $t_{80}$ :

$$
I\left(C_{f}, R, D, t_{80}\right)=\left\lceil\frac{D}{\left(C_{f}-C_{0}\right) R}\right\rceil\left(t_{80} \cdot \frac{\ln \left(1-C_{f}\right)}{\ln 0.2}+I_{\min }\right)
$$

Explanation: The equation is essentially number of stops $\cdot$ idle time per stop.

The number of stops is given by $\left\lceil\frac{D}{\left(C_{f}-C_{0}\right) R}\right\rceil$, or the total distance divided by the range possible with the current charge. It is rounded up to avoid a non-integer number of stops. The total time per stop is given by $t_{80} \cdot \frac{\ln \left(1-C_{f}\right)}{\ln 0.2}+I_{m i n}$, where $I_{\text {min }}$ is the baseline time lost by making a stop [4]. $t_{80} \cdot \frac{\ln \left(1-C_{f}\right)}{\ln 0.2}$ is derived from the general exponential approach equation $C_{f}=1-e^{-k t}$ given the point $\left(t_{80}, 0.8\right)$.

From all this, it is confirmed that waiting for $100 \%$ charge is highly disadvantageous, and in order to minimize wait time, trucks should be charged to a mid-range state.

The following graph shows an example of this minimum value, calculated using the range and charging speed of the Freightliner eCascadia [9], a middle-of-the-road electric semi-truck expected to hit the markets in 2021: 


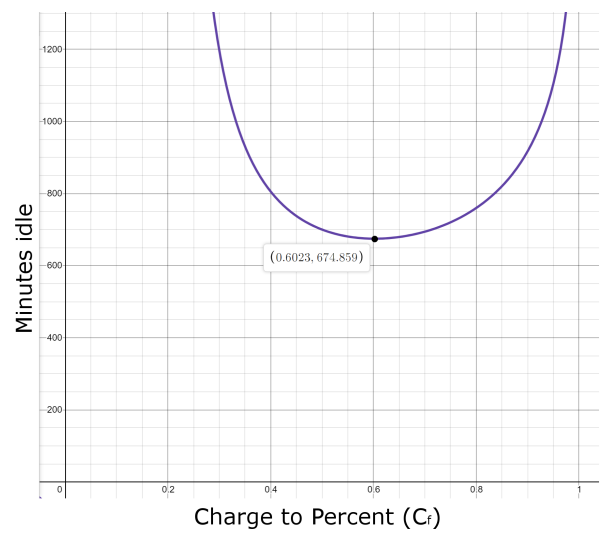

Figure 3: Freightliner eCascadia, $R=250 \mathrm{mi}, D=600 \mathrm{mi}, t_{80}=180 \mathrm{~min}$

\subsection{Executing the Model}

\subsubsection{Optimal Stations per Shipping Corridor}

To address the first problem stated in Part 2, in order to determine the number of stops needed along a given shipping corridor of distance $D$, we will use a Monte Carlo simulation with our formula for number of stops:

$$
\left\lceil\frac{D}{\left(C_{f}-C_{0}\right) R}\right\rceil
$$

We will hold $C_{0}$ constant at 0.2 (Assumption 2-4) and randomly vary $R$ and $C_{f}$ to test how frequently different trucks will need to stop.

- $R$ values will be normally distributed with $\mu=300$ and $\sigma=100$ conferring with the projected ranges of commercial electric semis [4].

- $C_{f}$ values will be uniformly distributed between 0.2 and 1 to simulate varied pit stop schedules used by drivers.

The Monte Carlo simulation was run for 1000 unique trucks, calculated for each of the given shipping corridors.

\subsubsection{Optimal Charging Stations per Truck Stop}

To address the second problem, another Monte Carlo simulation was used to determine the average number of trucks at a given station at any time, and thus, the optimal number of chargers for the station. The formula is as follows:

$$
\begin{aligned}
& \text { Avg. Trucks Present }=\sum_{i} \frac{I_{i}}{1440 \text { min }}, \\
& I_{i}=\frac{t_{80_{i}}}{\ln 0.2} \ln \left(1-C_{f}\right)-\ln \left(1-C_{0}\right),
\end{aligned}
$$


where $I_{i}$ is the time spent at the station by an individual truck derived from the exponential approach equation. Dividing by the number of minutes in a day provides the probability of encountering the truck at the station at any time.

To find this value, we ran the formula with 1000 different trucks with different idle times due to initial charge and charging speeds. So the Monte Carlo simulation was run varying the $C_{0}, C_{f}$, and $t_{80}$ values.

- $C_{0}$ values will be normally distributed with $\mu=0.6$ and $\sigma=0.125$, as before.

- $C_{f}$ values will be uniformly distributed between $C_{0}$ and 1 , to simulate varied charging schedules used by drivers.

- $t_{80}$ values will be uniformly distributed between 30 and 300 to account for the projected charging times of commercial electric semis [4].

\subsection{Results and Discussion}

Shown below are the results from the first Monte Carlo simulation, which found the optimal number of charging stops for each shipping corridor. It is worth noting that for all of them, it is almost exactly one stop every 90 minutes. This is unreasonable for diesel engines, but it makes sense given the nature of the electric batteries.
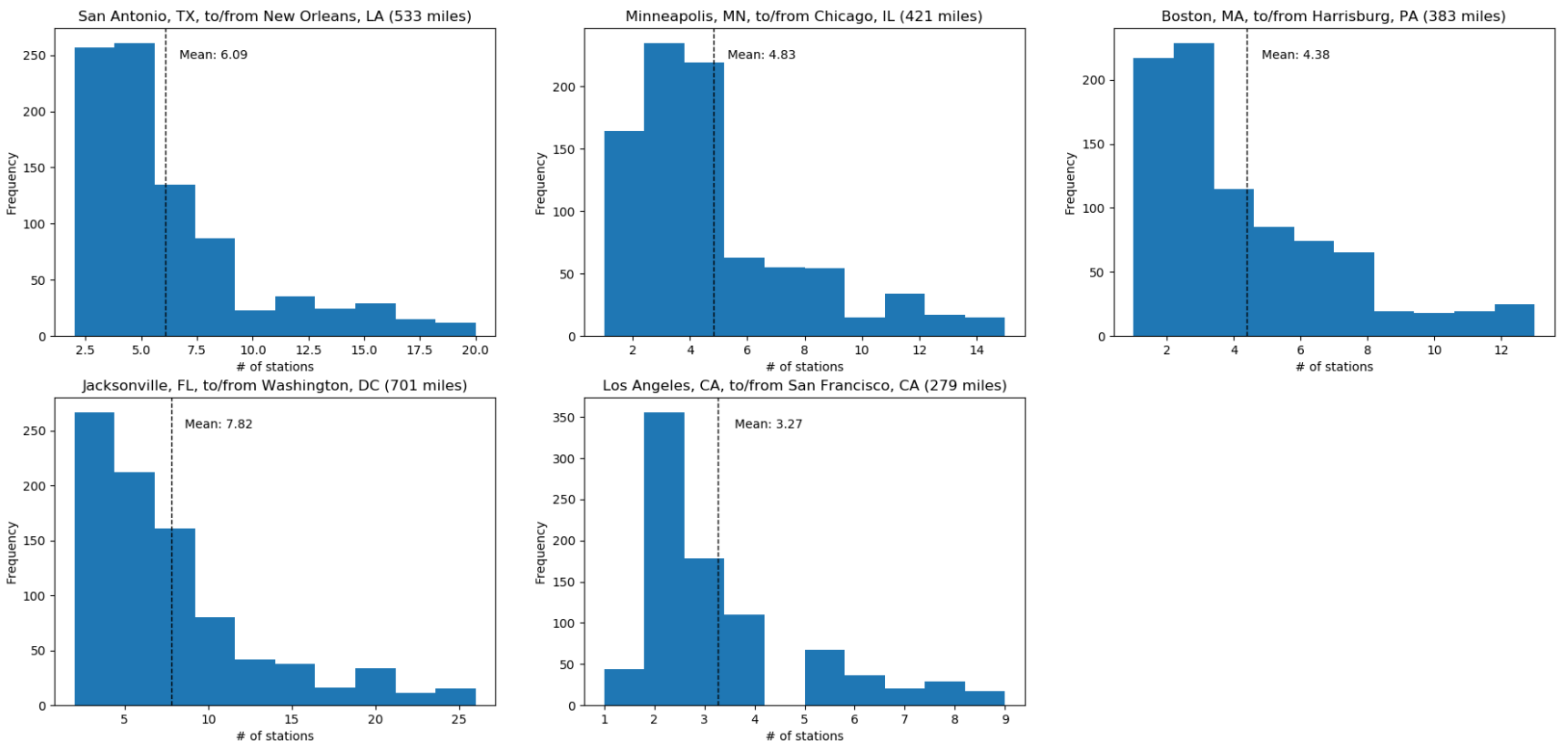

Figure 4: Monte Carlo simulation results, showing mean number of stops taken 
The following table shows the results from the second Monte Carlo simulation, which found the optimal number of charging stations at any given truck stop using its Annual Average Daily Truck Traffic:

\begin{tabular}{|c|l|l|l|}
\hline Corridor & $\begin{array}{l}\text { \# of Charging } \\
\text { Stations }\end{array}$ & $\begin{array}{l}\text { Miles per Charg- } \\
\text { ing Station }\end{array}$ & $\begin{array}{l}\text { \# of EVSE per } \\
\text { Station }\end{array}$ \\
\hline San Antonio to New Orleans & 6 & 87.5 & 17 \\
\hline Minneapolis to Chicago & 5 & 87.1 & 29 \\
\hline Boston to Harrisburg & 4 & 87.4 & 14 \\
\hline Jacksonville to Washington D.C. & 8 & 89.6 & 28 \\
\hline Los Angeles to San Francisco & 3 & 85.3 & 28 \\
\hline
\end{tabular}

It is worth comparing these numbers to a similar metric, the average number of Teslas at a supercharger, recently measured to be about 9 [5]. Since in this scenario, all semi-trucks are electric, the slightly increased numbers still make sense but are not unreasonable for a truck stop.

\subsubsection{Strengths and Weaknesses}

Due to the nature of the Monte Carlo simulations used, these models are fairly robust in that they account for a wide range of possibilities. The numbers, both constants and variables, were based on widely agreed-upon statistics.

However, the outputs - particularly for the first simulation - showed a larger-than-optimal standard deviation. There were a few significant outliers which required far more stops than the other trucks. We expect that this is an unrealistic product of our model, which could create an individual that would choose to refuel for a very small amount of time multiple times in a row.

Furthermore, a large part of our methodology was based on our Assumption 2-1, which holds that charging strictly conforms to the $1-e^{-k x}$ shape. Slight differences in real-world battery charging could possibly be amplified in the final results.

\section{I Like to Move It, Move It}

\subsection{Defining the Problem}

Create a model that ranks which trucking corridors should begin development of charging stations first and use this model to rank the five trucking corridors from the previous part.

\subsection{Assumptions}

3-1. The cost per mile of Semi Shipping is $\$ 2.75$.

- Justification: This is the current middle-of-the-market average value. [7]. 
3-2 The cost is $\$ 22,626$ to install one DCFC station.

- Justification: The mean cost of installing a DCFC Station was 22,626 based on data published by the U.S. Department of Energy [6].

3-3. $50 \%$ trucks using the corridors are long-haul shipments.

- This statistic is found in the Keep on Trucking Information Sheet provided by the MathWorks Math Modeling Challenge 2020 [2].

\subsection{Variables Used}

\begin{tabular}{|c|c|c|c|}
\hline Symbol & Definition & Units & Value \\
\hline$F$ & Cost per Mile of Semi Shipping & $\$ /$ Mile & 2.75 \\
\hline$V$ & Net value per minute of Semi Shipping & $\$ /$ Mile & $\ldots$ \\
\hline$T_{\text {avg }}$ & Average Daily Truck Traffic of the route & Trucks/Day & $\ldots$ \\
\hline$S$ & Total Cost of Charging Station Installations & $\$$ & $\ldots$ \\
\hline$X$ & Importance Score for each corridor & - & $\ldots$ \\
\hline$E$ & Community Environmental Measures & - & $\ldots$ \\
\hline$G$ & Increase in GDP from Trucking Time & $\$ /$ Year & $\ldots$ \\
\hline
\end{tabular}

\subsection{Developing the Model}

In order to rank which trucking corridors to develop first, we will consider three metrics:

1. Economic Growth - i.e. GDP increase,

2. Environmental considerations, and

3. Cost to implement the necessary infrastructure.

All metrics for each of the five corridors will be normalized, equally weighted, and combined into a final importance score for each corridor, $X$, as follows:

$$
X=G_{\text {norm }}+E_{\text {norm }}+S_{\text {norm }}
$$

For the first metric, economic growth, $G$, was calculated by the money saved by decreased idle charging times of DCFC chargers. $V$, the dollar value of an on-route semi's time, was calculated using the average cost of semi shipping, $F=2.75 / \mathrm{mi}$ (Assumption $3-1$ ), and the average number of trucks using the corridor, $T_{\text {avg }}$. $T_{\text {avg }}$ was found by taking $50 \%$ of the average value of the Annual Average Daily Truck Traffic (AADTT) for each corridor (per Assumption 3-3).

For each corridor, $V$ was found by the following formula:

$$
V=\frac{F D}{D \cdot \frac{1 m i n}{1 m i}+I},
$$


where $D$ is the length of the corridor and $I$ is the total idle time while charging, calculated using recharge and range values from the Freightliner eCascadia.

The $T_{\text {avg }}$ values for each of the five corridors are shown below:

\begin{tabular}{|c|c|}
\hline Corridor & $T_{\text {avg }}$ \\
\hline San Antonio to New Orleans & $7,144.05$ \\
\hline Minneapolis to Chicago & $8,010.75$ \\
\hline Boston to Harrisburg & $4,646.43$ \\
\hline Jacksonville to Washington D.C. & $4,757.54$ \\
\hline Los Angeles to San Francisco & $6,987.43$ \\
\hline
\end{tabular}

Thus, the total economic value generated is given by

$$
G=V \cdot T_{a v g} .
$$

For the second metric, E, environmental considerations were based off of number of state actions on electric vehicles found in a report conducted by the NC Clean Energy Technology Center [8]. Actions consisted of setting targets for zero-emission vehicle, plans for transportation electrification plans, and exemptions of charging stations from public utility regulation. Number of actions was categorized into five levels:

\begin{tabular}{|c|c|}
\hline Level & \# of Actions \\
\hline 0 & none \\
\hline 1 & 1 to 2 \\
\hline 2 & 3 to 5 \\
\hline 3 & 6 to 9 \\
\hline 4 & 10 or more \\
\hline
\end{tabular}

Table 6: Environmental Consideration Levels

Environmental considerations was considered as communities who were more environmentally conscious and had more incentives and actions regarding electric vehicles would likely be more motivated to support development of charging stations. The environmental consideration score per trucking corridor was calculated by averaging the levels of all the states and are listed in Table 7.

For the third metric, the cost of charging station installations, $S$, is based on the cost to install one DCFC station and the solution to Part 2, as follows:

$$
\begin{aligned}
& \text { Avg. Trucks Present }=\text { chargers } / \text { station } \\
& S=\$ 22,626 \cdot \text { chargers } / \text { station } \cdot \text { stations }
\end{aligned}
$$

These metrics are then normalized through the use of min-max normalization to weight the values the same and be able to add them together in determining the Importance Score. The formula is as follows:

$$
\text { Normalized Value }=\frac{x_{i}-x_{\min }}{x_{\max }-x_{\min }}
$$




\subsection{Executing the Model}

\begin{tabular}{|c|c|c|c|}
\hline Trucking Corridors & E & S & G \\
\hline San Antonio to New Orleans & 3 & $\$ 2,692,494$ & $\$ 9,644$ \\
\hline Minneapolis to Chicago & 3.67 & $\$ 3,280,770$ & $\$ 10,814$ \\
\hline Boston to Harrisburg & 3.8 & $\$ 1,583,820$ & $\$ 6,272$ \\
\hline Jacksonville to Washington D.C. & 2.5 & $\$ 5,068,224$ & $\$ 6,421$ \\
\hline Los Angeles to San Francisco & 4 & $\$ 2,534,112$ & $\$ 9,432$ \\
\hline
\end{tabular}

Table 7: Importance Score for Trucking Corridors

\begin{tabular}{|c|c|c|c|c|}
\hline Trucking Corridors & E & S & G & X \\
\hline San Antonio to New Orleans & 0.33 & 0.32 & 0.74 & 1.39 \\
\hline Minneapolis to Chicago & 0.78 & 0.49 & 1 & 2.27 \\
\hline Boston to Harrisburg & 0.87 & 0 & 0 & 0.87 \\
\hline Jacksonville to Washington D.C. & 0 & 1 & 0.03 & 1.03 \\
\hline Los Angeles to San Francisco & 1 & 0.27 & 0.70 & 1.97 \\
\hline
\end{tabular}

Table 8: Normalized Importance Score for Trucking Corridors

\subsection{Results and Discussion}

The results showed that the Minneapolis to Chicago corridor should be targeted first followed by Los Angeles to San Francisco then San Antonio to New Orleans then Jacksonville to D.C. then Boston to Harrisburg.

\subsubsection{Strengths and Weaknesses}

The strength of our model is that we consider three different variables.

A weakness of the model is that there are other variables that could be considered in the making of the model such as electric vehicle usage in the states and how much each route is used. A second weakness is that the cost has a high standard deviation, which could greatly affect the results of the model. 


\section{References}

[1] https://www.iea.org/news/iea-study-unveils-key-role-for-trucks-in-global-oil-demandgrowth.

[2] https://m3challenge.siam.org/node/478.

[3] https://www.mylittlesalesman.com/semi-trucks.

[4] https://nacfe.org/future-technology/amping-up-charging-infrastructure-for-electrictrucks.

[5] https://electrek.co/2019/12/19/tesls-supercharger-reaches-15000-chargers-best-assets.

[6] https://www.energy.gov/eere/vehicles/fact-910-february-1-2016-study-shows-averagecost-electric-vehicle-charger.

[7] https://truckerpath.com/blog/truck-freight-rates/.

[8] https://nccleantech.ncsu.edu/wp-content/uploads/2019/08/Q2-

19_EV_execsummary_Final.pdf.

[9] https://freightliner.com/e-mobility/.

[10] https://hdstruckdrivinginstitute.com/semi-trucks-numbers/.

[11] https://electrek.co/2019/10/23/tesla-semi-electric-truck-production-limited-volume$2020 /$.

[12] https://spectrum.ieee.org/cars-that-think/transportation/advanced-cars/teslalaunches-an-electric-semi-truckand-a-new-sports-car.

[13] https://www.powerstream.com/LLLF.htm.

[14] https://www.dmcinfo.com/latest-thinking/blog/id/9969/everything-you-need-toknow-about-ev-battery-and-bms-testing-in-validation-and-production-scenarios.

[15] https://www.bizjournals.com/sanjose/news/2017/11/27/tesla-semi-truck-price-costfuel-tsla.html.

[16] https://www.theverge.com/2017/11/16/16655890/tesla-semi-truck-2017-freightweight-fuel-range.

[17] https://m3challenge.siam.org/sites/default/files/uploads/semi_production_and_use.xlsx. 


\section{Code Used}

\section{Part 1}

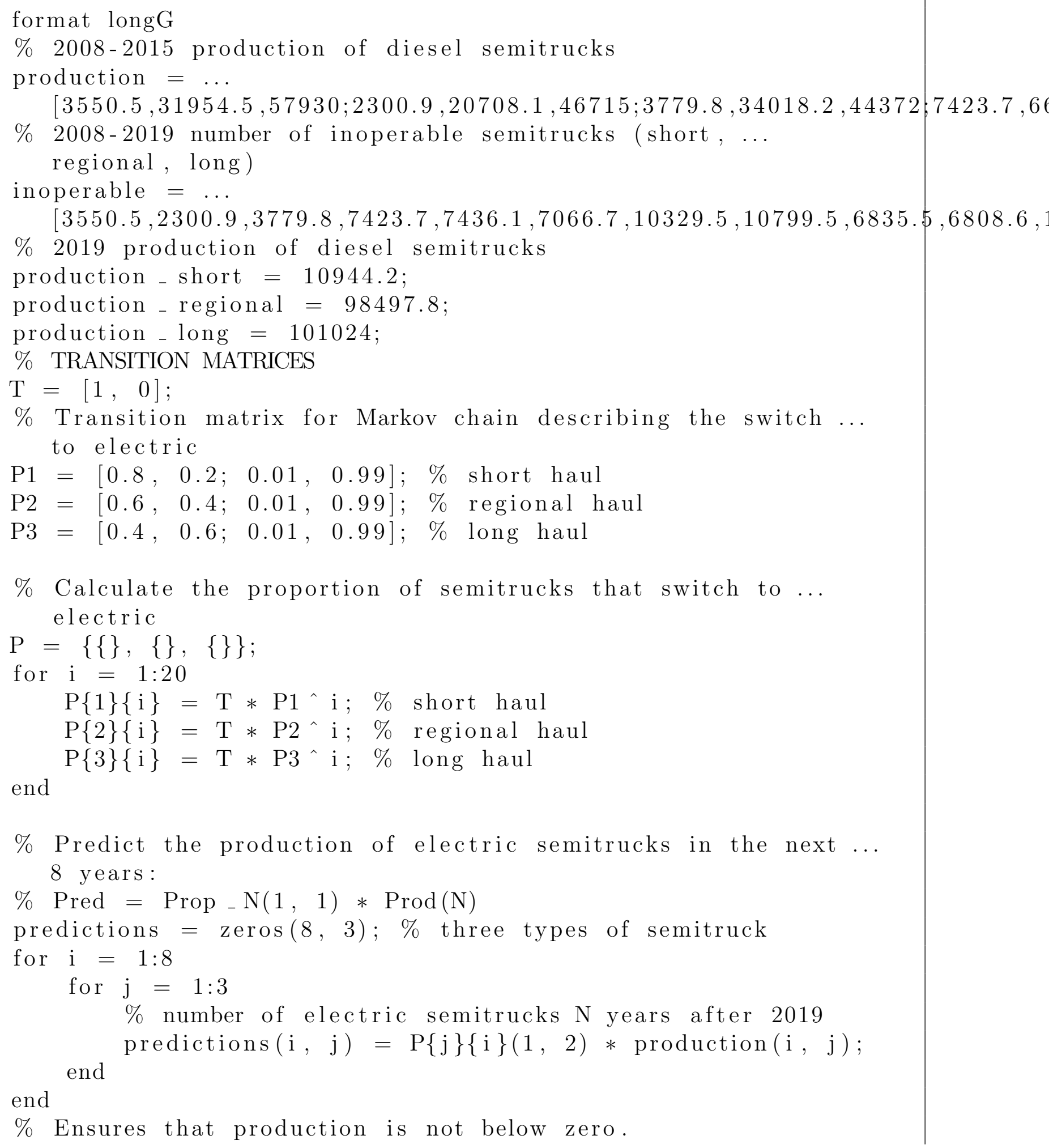


predictions $($ predictions $; 0)=0$

\% Converts $\mathrm{P}$ to an array of the proportions.

Prop = cell2mat (cellfun(@cell2mat, cellfun(@transpose, P,...

'UniformOutput', false), 'UniformOutput', false));

$\%$ Calculate the number of

$\%$ short haul semitrucks after 5 years - I $(1,1) *$ Prop - 5

short $-5=\operatorname{sum}($ inoperable $(1: 5,1) . * \operatorname{Prop}(1: 5,1: 2)) \% \# \ldots$

ok $i *$ NOPTS $i$

$\%$ short haul semitrucks after 10 years - I (1, 2) * Prop - 10

short $-10=\operatorname{sum}($ inoperable $(1: 10,1) . * \operatorname{Prop}(1: 10,1: 2))$

$\%$ short haul semitrucks after 20 years - I $(1,3) *$ Prop -20

short $-20=\operatorname{sum}($ inoperable $(1: 12,1) . * \operatorname{Prop}(1: 12,1: 2))$

$\%$ regional haul semitrucks after 5 years - I $(2,1) *$ Prop - 5

regional $-5=\operatorname{sum}($ inoperable $(1: 5,2) * \operatorname{Prop}(1: 5,3: 4)$ )

$\%$ regional haul semitrucks after 10 years - I $(2,2) *$ Prop - 10

regional $-10=\operatorname{sum}($ inoperable $(1: 10,2) * \operatorname{Prop}(1: 10,3: 4))$

$\%$ regional haul semitrucks after 20 years - I $(2,3) *$ Prop -20

regional $-20=\operatorname{sum}($ inoperable $(1: 12,2) . * \operatorname{Prop}(1: 12,3: 4))$

$\%$ long haul semitrucks after 5 years - I $(3,1) *$ Prop -5

long $-5=\operatorname{sum}($ inoperable $(1: 5,3) * \operatorname{Prop}(1: 5,5: 6))$

$\%$ long haul semitrucks after 10 years - I $(3,2) *$ Prop - 10

long $-10=\operatorname{sum}($ inoperable $(1: 10,3) . * \operatorname{Prop}(1: 10,5: 6))$

$\%$ long haul semitrucks after 20 years - I $(3,3) *$ Prop _ 20

long $-20=\operatorname{sum}($ inoperable $(1: 12,3) * \operatorname{Prop}(1: 12,5: 6))$

$\%$ Diagram of the Markov chain

$\mathrm{P}=[0.8,0.2$

$0.01,0.99]$

$\mathrm{mc}=\operatorname{dtmc}(\mathrm{P})$;

figure ;

graphplot (mc); 


\section{Part 2}

\#pylint: disable=bad-continuation, invalid-name, multiple-imports, redefined-outer-name

Created by Team \#13343 1 March 2020

This is the Monte Carlo method for Part 2 . " " "

import math, random

import numpy as np

import matplotlib.pyplot as plt

import matplotlib

matplotlib.rcParams ["figure.figsize"] = [22, 10]

initial $=0.2 \#$ initial state of charge

epsiton $=10 \mathrm{e}-6$ \# avoid division by zero

$k=1.5$ \# Tukey's fence "outlier" constant

nos $=[$ [ \# number of stations array

def tukeys_fences(arr, q_1, q_3):

"1" "Tukey's fences for defining outliers. " "n

iqr $=q_{-} 3-q_{-} 1$ \# interquartile range

arr_t = arr \# temporary array to avoid iteration problems

for $i$ in arr: \# iterate over array

if $i<\left(q_{-} 1-k *\right.$ iqr $)$ or $i>\left(q_{-} 3+k *\right.$ iqr $)$ : \# Tukey's fences

$\operatorname{arr} r_{-}=n p \cdot d e l e t e\left(\operatorname{arr} r_{-}, n_{p} \cdot \operatorname{argwhere}\left(\operatorname{arr} r_{-} t==i\right)\right)$ \# exclude outliers

return arr_t \# array without outliers

def number_of_stations(final, erange, distance):

" "The number of stations needed along a given route." " "

return math.ceil(distance / ((final - initial) * erange + epsilon)) \# see 2.4

def truck_time_at_station(initial, final, t80):

"" "A truck's time at a station." ""

return $($ t80 / math. $\log (0.2)) *($ math. $\log (1$ - final $)$ - math. $\log (1$ - initial $))$ \# see 2.5

\# Substitute proper distances for corridors

distances $=[533$, \# SanTX_NewLA

421, \# MinMN_ChiIL

383, \# BosMA_HarPA

701, \# JacFL_WasDC

279] \# LosCA_SanCA

corridors $=$ [ "San Antonio, TX, to/from New Orleans, LA",

"Minneapolis, MN, to/from Chicago, IL",

"Boston, MA, to/from Harrisburg, PA",

"Jacksonville, FL, to/from Washington, DC",

"Los Angeles, CA, to/from San Francisco, CA"]

corridors $=$ [corridors $[i]+"("+\operatorname{str}(x)+"$ miles $) "$ for $i, x$ in enumerate(distances $)]$

AADT_max $=[168267$, \# SanTX_NewLA

305100, \# MinMN_ChiIL

144200 , \# BosMA HarPA

264000, \# JacFL_WasDC

304000] \# LosCA_SanCA

\# Initialize random convoy of 1,000 all-electric trucks

\# Discrete uniform distribution

final_mcl $=$ np.random. uniform(0.2 + epsilon, 1.0, 1000)

\# Discrete normal distribution

erange $m c=n p$. random.normal(300, 100, 1000)

\# Exclude values outside the domain

erange_mc [erange_mc $<0]=0$

\# Vectorize functions to parallelize them

vnos $=$ np.vectorize(number_of_stations)

vttas $=$ np.vectorize(truck_time_at_station)

\# Evaluate vectorized functions for random samples

for d in distances: \# iterate over array of distances for corridors

array_vnos $=\operatorname{vnos}\left(f i n a l \_m c 1\right.$, erange_mc, $d$ )

Q1 = np.percentile(array_vnos, 25, interpolation="midpoint") \# first quartile

Q3 = np.percentile(array_vnos, 75, interpolation="midpoint") \# third quartile

nos.append(tukeys_fences(array_vnos, Q1, Q3)) \# exclude outliers and append results to nos

for AADT in AADT_max: \# iterate over array of Annual Average Daily Traffic data

t80 $\mathrm{mc}=\mathrm{np}$.random.uniform(30, 300, AADT)

initial_mc $=n p$. random.normal $(0.6,0.125$, AADT $)$

initial_mc[np.logical_or (initial_mc $<0$, initial_mc $>=1)]=0$

final_mc2 $=\left[\operatorname{random} . \operatorname{uniform}_{(x, 1.0)}\right.$ for $x$ in initial_mc.tolist( $\left.)\right]$

array_ttas $=$ vttas $($ initial_mc, final_mc2, t80_mc) [np.argwhere(initial_mc $<=0.2)$ ]

number_of_chargers_station = math.ceil(sum(array_ttas)[0] / 1440)

means $=[\operatorname{nos}[\bar{i}] . m e a n()$ for $i$ in $\operatorname{range}(\operatorname{len}(\operatorname{nos}))]$ \# evaluate mean for all corridors

fig, axs = plt.subplots $(2,3)$

for $i$, ax in np.ndenumerate(np.delete(axs, -1)):

ax.hist (nos [i[0]])

ax.axvline(means[i[0]], color="k", linestyle="dashed", linewidth=1)

ax.text(means[i[0] $] * 1.1$, ax.get_ylim()[1]*0.9, "Mean: $\{: .2 f\} "$ format(means $[i[0]]))$

ax.set_title(corridors[i[0]])

ax.set_xlabel("\# of stations")

ax.set_ylabel("Frequency")

axs[1, 2].axis("off") 